\title{
Research Article \\ Delay-Dependent Asymptotic Stability of Cohen-Grossberg Models with Multiple Time-Varying Delays
}

\author{
Xiaofeng Liao and Songtao Guo \\ Received 4 November 2006; Accepted 1 March 2007
}

Dynamical behavior of a class of Cohen-Grossberg models with multiple time-varying delays is studied in detail. Sufficient delay-dependent criteria to ensure local and global asymptotic stabilities of the equilibrium of this network are derived by constructing suitable Lyapunov functionals. The obtained conditions are shown to be less conservative and restrictive than those reported in the known literature. Some numerical examples are included to demonstrate our results.

Copyright (c) 2007 X. Liao and S. Guo. This is an open access article distributed under the Creative Commons Attribution License, which permits unrestricted use, distribution, and reproduction in any medium, provided the original work is properly cited.

\section{Introduction}

A large class of neural networks, which can function as stable content addressable memories or CAMs [1, 2], had been proposed by Cohen and Grossberg. These Cohen-Grossberg networks were designed to include additive neural networks, later studied by Hopfield $[3,4]$, and shunting neural networks. In the original analysis, Cohen and Grossberg assumed that the weight matrix was symmetric. Meanwhile, the activation functions are assumed to be continuous, differentiable, monotonically increasing, and bounded, such as the sigmoid-type function. Usually, such systems have been investigated under the assumption of asymmetric connection weight and nonmonotonic activation function. However, monotonicity and differentiability of activation functions come form the experimental results of brain sciences, moreover, they have very strong biological background. On the other hand, realistic modeling of many large neural networks with nonlocal interaction inevitably requires connection delays to be taken into account, since they naturally arise as a consequence of finite information transmission and processing speeds among the neurons. 
It is also important to incorporate time delay into the model equations of the network such as delayed cellular neural network, which can be used to solve problems like the processing of moving images $[5,6]$. Ye et al. [7] introduced discrete delays into the Cohen-Grossberg model. Furthermore, their global stability needed to satisfy the requirements that the connection should possess certain amount of symmetry and the discrete delays were sufficiently small.

For the delayed Hopfield networks [7-14], cellular neural networks [5, 6], as well as BAM networks [15-18], some delay-independent criteria for the global asymptotic stability are established without assuming the monotonicity and the differentiability of the activation functions and also the symmetry of the connection. Wang and Zou [19] also studied the Cohen-Grossberg model with time delays. The global stability criteria of this type of neural networks were also obtained by constructing appropriate Lyapunov functionals, or Lyapunov functions combined with the Rezumikhin technique. All of these criteria are independent of the magnitudes of the delays, and therefore the delays are harmless in a network satisfying one of the criteria. Actually, the global exponential stability implies global asymptotic stability, and so the results leading to global exponential stability can provide relevant estimates on how fast such networks perform during realtime computations. Furthermore, Liao et al. [20,21] studied this problem.

Generally, the stability criteria for time-delay systems can be classified into two categories, namely delay-independent criteria and delay-dependent criteria, depending on whether they contain the delay argument as a parameter. There have been a number of significant developments in searching the stability criteria for systems with constant delays $[4,6,7,9,11,12,14-17]$. Only a few of them are for neural networks with distributed delays; see, for instance, $[1-5,8,10,18]$. To the best of the authors' knowledge, the delaydependent criteria in the case of the delayed Cohen-Grossberg model are little studied yet. In this paper, we will present some new local and global asymptotic stabilities of the equilibrium of Cohen-Grossberg models with mulitiple delays. Our results essentially show that the equilibrium of the network remains globally asymptotically stable when the time delays are small enough. In order to prove our results, we construct the suitable Lyapunov functionals.

In this paper, the amplification functions need to be continuous, positive, and bounded. However, the self-signal functions are not assumed to be differentiable, but only need to satisfy condition $\left(\mathrm{H}_{2}\right)$, as stated in the next section. At the same time, we do not confine ourselves to the symmetric connections. The rest of this paper is organized as follows. In Section 2, the Cohen-Grossberg neural network with time-varying delays and some preliminary analyses are given. By constructing Lyapunov functionals, some global exponential stability criteria for the network are presented in Section 3. Finally, numerical example is given to illustrate our results and some conclusions are drawn in Section 4 .

\section{Some preliminaries and network models}

We consider Cohen-Grossberg neural networks with multiple time-varying delays, described by equations of theform 


$$
\dot{u}_{i}(t)=-a_{i}\left(u_{i}(t)\right)\left[b_{i}\left(u_{i}(t)\right)-\sum_{k=0}^{K} \sum_{j=1}^{n} t_{i j}^{(k)} f_{j}\left(u_{j}\left(t-\tau_{k}(t)\right)\right)+I_{i}\right], \quad i=1,2, \ldots, n,
$$

where $u_{i}$ denotes the state variable associated with the $i$ th neuron, the function $a_{i}$ represents an amplification function, and $b_{i}$ is an arbitrary function; however we will require that $b_{i}$ be sufficiently well behaved to keep the solutions of (2.1) bounded. The $t_{i j}^{(k)}$ 's denote the interconnections which are associated with delay $\tau_{k}(t), \tau_{k}(t)$ denotes the $k$ th time delay for $k=0,1,2, \ldots, K$ such that $0=\tau_{0}<\tau_{1}<\cdots<\tau_{k}$.

System (2.1) is said to be globally stable if for any solution $u(t), \lim _{t \rightarrow \infty} u(t)$ exists. For the definitions of stability and asymptotic stability of an equilibrium of (2.1), refer to any of several standard texts (see, e.g., [22]).

In this paper, we assume that the Cohen-Grossberg neural networks (2.1) satisfy the following assumptions.

$\left(\mathrm{H}_{1}\right)$ The function $a_{i}$ is bounded, positive, and continuous.

$\left(\mathrm{H}_{2}\right)$ The function $b_{i}$ is continuous, and there exist positive constants $\underline{B_{i}}$ and $\overline{B_{i}}, i=$ $0,1,2, \ldots, n$, such that

$$
\begin{gathered}
0<\underline{B_{i}} \leq \frac{b_{i}\left(x_{i}\right)-b_{i}\left(y_{i}\right)}{x_{i}-y_{i}} \leq \overline{B_{i}}, \quad \text { for } x_{i} \neq y_{i}, i=1,2, \ldots, n, \\
\lim _{u_{i} \rightarrow+\infty} b_{i}\left(u_{i}\right)=+\infty, \quad \lim _{u_{i} \rightarrow-\infty} b_{i}\left(u_{i}\right)=-\infty
\end{gathered}
$$

$\left(\mathrm{H}_{3}\right) f_{j} \in C^{1}(\mathbb{R}, \mathbb{R})$ is a sigmoidal function (so that $s_{j}^{\prime}\left(x_{j}\right) \triangleq d s_{j}\left(x_{j}\right) / d x_{j}>0$, $\lim _{x_{j} \rightarrow+\infty} f_{j}\left(x_{j}\right)=1, \lim _{x_{j} \rightarrow-\infty} f_{j}\left(x_{j}\right)=-1$, and $\left.\lim _{\left|x_{j}\right| \rightarrow \infty} s_{j}^{\prime}\left(x_{j}\right)=0\right)$.

$\left(\mathrm{H}_{4}\right) \tau_{k}:[0,+\infty) \rightarrow[0,+\infty)$ is continuous and $0 \leq \tau_{k}(t) \leq \bar{\tau}$.

The initial condition for system (2.1) is given as follows:

$$
u_{j}(s)=\phi_{j}(s), \quad s \in[-\bar{\tau}, 0]
$$

Lemma 2.1. If assumption $\left(H_{1}\right)-\left(H_{4}\right)$ are satisfied for system (2.1)-(2.3), then any solution of (2.1) and (2.3) is bounded.

Proof. We only need to consider system (2.1). We know by $\left(\mathrm{H}_{1}\right)-\left(\mathrm{H}_{4}\right)$ that the terms $f_{j}\left(u_{j}(t)\right)$ and $f_{j}\left(u_{j}\left(t-\tau_{k}(t)\right)\right)$ are bounded for all $j=1,2, \ldots, n$. Furthermore, since $\lim _{u_{i} \rightarrow+\infty} b_{i}\left(u_{i}\right)=+\infty$ and $\lim _{u_{i} \rightarrow-\infty} b_{i}\left(u_{i}\right)=-\infty$, there must exist $M>0$ such that

$$
b_{i}\left(u_{i}(t)\right)-\sum_{k=0}^{K} \sum_{j=1}^{n} t_{i j}^{(k)} f_{j}\left(u_{j}\left(t-\tau_{k}(t)\right)\right)+I_{i}>0
$$

whenever $u_{i}(t) \geq M$ and

$$
b_{i}\left(u_{i}(t)\right)-\sum_{k=0}^{K} \sum_{j=1}^{n} t_{i j}^{(k)} f_{j}\left(x_{j}\left(t-\tau_{k}(t)\right)\right)+I_{i}<0
$$

whenever $u_{i}(t) \leq-M$ for all $i=1,2, \ldots, n$. Since $a_{i}\left(u_{i}(t)\right)$ is positive by $\left(\mathrm{H}_{1}\right)$, it can be concluded that for any solution $u(t)$ of system $(2.1), \dot{u}_{i}(t)<0$ whenever $u_{i}(t) \geq M$ and 
$\dot{u}_{i}(t)>0$ whenever $u_{i}(t) \leq-M$ for all $i=1,2, \ldots, n$. We may assume that for the initial condition $\phi_{j}(s),\left|\phi_{j}(s)\right|<M$, otherwise we just pick a larger $M$. Thus we can conclude that $\left\|u_{i}(t)\right\| \leq M$ for all $t \geq 0$ and all $i=1,2, \ldots, n$.

It is not difficult to show that under $\left(\mathrm{H}_{1}\right)-\left(\mathrm{H}_{4}\right)$, the solution of $(2.1)$ satisfying the initial condition (2.3) exists on $\mathbb{R}_{+} \equiv[0,+\infty)$ (see, e.g., $[22,23]$ ). Actually, note that from Lemma 2.2 , it is clear that the solution of (2.1) is also unique.

It is also easy to show that $(2.1)$ has always an equilibrium $u_{j}^{*}, i=1,2, \ldots, n$. That is, there exist $u_{j}^{*}, i=1,2, \ldots, n$, such that

$$
b_{i}\left(u_{i}^{*}\right)=\sum_{k=0}^{K} \sum_{j=1}^{n} t_{i j}^{(k)} f_{j}\left(u_{j}^{*}\right)+I_{i}, \quad i=1,2, \ldots, n .
$$

By using the strict monotonicity property of $b_{i}$, there exist positive numbers $\overline{b_{i}}>0$, $i=1,2, \ldots, n$, such that

$$
b_{i}\left(u_{i}^{*}\right)=\overline{b_{i}} u_{i}^{*}, \quad i=1,2, \ldots, n \text {. }
$$

Thus,

$$
u_{i}^{*}=\bar{b}_{i}^{-1}\left\{\sum_{k=0}^{K} \sum_{j=1}^{n} t_{i j}^{(k)} f_{j}\left(u_{j}^{*}\right)+I_{i}\right\}, \quad i=1,2, \ldots, n .
$$

In fact, let us consider the map $P=\left(P_{1}, P_{2}, \ldots, P_{n}\right)$ on the compact convex set $\Omega$, where

$$
\begin{gathered}
P_{i}\left(u_{1}, u_{2}, \ldots, u_{n}\right)=\bar{b}_{i}^{-1}\left\{\sum_{k=0}^{K} \sum_{j=1}^{n} t_{i j}^{(k)} f_{j}\left(u_{j}^{*}\right)+I_{i}\right\}, \quad i=1,2, \ldots, n, \\
\Omega=\left\{\left(u_{1}, u_{2}, \ldots, u_{n}\right)|| u_{i} \mid \leq N_{i 0}\right\}, \\
N_{i 0}=\frac{\sum_{k=0}^{K} \sum_{j=1}^{n}\left|t_{i j}^{(k)}\right| F_{j}+\left|I_{i}\right|}{\left|\bar{b}_{i}\right|}, \quad\left|f_{j}\left(u_{j}^{*}\right)\right| \leq F_{j}, \quad j=1,2, \ldots, n .
\end{gathered}
$$

It follows from $\left(\mathrm{H}_{1}\right)$ that $P$ is a continuous map $\Omega$ into itself. Thus, it follows from Brouwer's fixed point theorem (see, e.g., [22]) that $P$ has at least one fixed point $\left(u_{1}^{*}\right.$, $\left.u_{2}^{*}, \ldots, u_{n}^{*}\right)$ in $\Omega$, that is,

$$
\left(u_{1}^{*}, u_{2}^{*}, \ldots, u_{n}^{*}\right)=P\left(u_{1}^{*}, u_{2}^{*}, \ldots, u_{n}^{*}\right) .
$$

This shows that $\left(u_{1}^{*}, u_{2}^{*}, \ldots, u_{n}^{*}\right)$ satisfies $(2.6)$. 
Lemma 2.2 is immediate.

LEMMA 2.2. If $\left(H_{1}\right)-\left(H_{4}\right)$ are satisfied, then for any solution of (2.1),

$$
\limsup _{t \rightarrow \infty}\left|u_{i}(t)\right| \leq N_{i}\left(\leq N_{i 0}\right), \quad i=1,2, \ldots, n,
$$

where the positive constants $N_{i}, i=1,2, \ldots, n$, satisfy

$$
\begin{gathered}
N_{i}=\frac{\sum_{k=0}^{K} \sum_{j=1}^{n}\left|t_{i j}^{(k)}\right| \bar{f}_{i}\left(N_{i}\right)+\left|I_{i}\right|}{\left|\overline{b_{i}}\right|}, \\
\overline{f_{i}}\left(N_{i}\right)=\max \left\{f_{i}\left(N_{i}\right),-f_{i}\left(-N_{i}\right)\right\}, \quad i=1,2, \ldots, n .
\end{gathered}
$$

Proof. It is clear from $\left(\mathrm{H}_{1}\right)-\left(\mathrm{H}_{4}\right)$ and $(2.1)$ that

$$
\lim _{t \rightarrow \infty} \sup \left|u_{i}(t)\right| \leq N_{i 0}, \quad i=1,2, \ldots, n
$$

Thus, for sufficiently small $\eta>0$ and sufficiently large $T_{0}>0$, such that for $t \geq T_{0}$,

$$
\left|u_{i}(t-\tau)\right| \leq N_{i 0}+\eta, \quad i=1,2, \ldots, n,
$$

which together with $\left(\mathrm{H}_{3}\right)$ and (2.1) yield that for $t \geq T_{0}$,

$$
\dot{u}_{i}(t) \leq a_{i}\left(u_{i}(t)\right)\left[-\left|d_{i}\left(u_{i}(t)\right)\right|+\sum_{k=0}^{K} \sum_{j=1}^{n}\left|t_{i j}^{(k)}\right| \overline{f_{j}}\left(N_{i 0}+\eta\right)+\left|I_{i}\right|\right], \quad i=1,2, \ldots, n .
$$

Note that one can take $\eta \rightarrow 0$ as $t \rightarrow+\infty$, we have

$$
\limsup _{t \rightarrow \infty}\left|u_{i}(t)\right| \leq N_{i 1}, \quad i=1,2, \ldots, n,
$$

where

$$
N_{i 1}=\frac{\sum_{k=0}^{K} \sum_{j=1}^{n}\left|t_{i j}^{(k)}\right| \overline{f_{i}}\left(N_{i 0}\right)+\left|I_{i}\right|}{\left|\overline{b_{i}}\right|} \leq N_{i 0}, \quad i=1,2, \ldots, n
$$

By repeating the above procedure, we can obtain positive sequences $\left\{N_{i, k}\right\}$ such that

$$
\begin{gathered}
N_{i, k+1}=\frac{\sum_{k=0}^{K} \sum_{j=1}^{n}\left|t_{i j}^{(k)}\right| \overline{f_{i}}\left(N_{i, k}\right)+\left|I_{i}\right|}{\left|\bar{b}_{i}\right|} \leq N_{i, k}, \quad i=1,2, \ldots, n, \\
\limsup _{t \rightarrow \infty}\left|u_{i}(t)\right| \leq N_{i, k}, \quad i=1,2, \ldots, n, k=1,2, \ldots
\end{gathered}
$$


Let $N_{i}$ denote the limits of $\left\{N_{i, k}\right\}$ as $k \rightarrow+\infty$, respectively. Then, we have

$$
\begin{gathered}
N_{i}=\left\{\frac{\sum_{k=0}^{K} \sum_{j=1}^{n}\left|t_{i j}^{(k)}\right| \overline{f_{i}}\left(N_{i, k}\right)+\left|I_{i}\right|}{\left|\overline{b_{i}}\right|}\right\}, \quad i=1,2, \ldots, n, \\
\lim _{t \rightarrow \infty} \sup \left|u_{i}(t)\right| \leq N_{i} .
\end{gathered}
$$

This shows that Lemma 2.2 holds.

By Lemma 2.2, we see that for any sufficiently small positive constant $\varepsilon$, there exists a sufficiently large time, $T=T(\varepsilon)>0$, such that for $t \geq T$,

$$
\left|u_{i}(t)\right| \leq N_{i}+\varepsilon, \quad i=1,2, \ldots, n \text {. }
$$

Define positive constants $p_{i, \varepsilon}$ and $q_{i, \varepsilon}, i=1,2, \ldots, n$, as follows:

$$
p_{i, \varepsilon} \equiv \min _{-\left(N_{i}+\varepsilon\right) \leq w \leq N_{i}+\varepsilon} f_{i}^{\prime}(w) \leq \max _{-\left(N_{i}+\varepsilon\right) \leq w \leq N_{i}+\varepsilon} g_{i}^{\prime}(w) \equiv q_{i, \varepsilon}, \quad i=1,2, \ldots, n .
$$

Let $p_{i}$ and $q_{i}, i=1,2, \ldots, n$, denote the limits of $p_{i \varepsilon}$ and $q_{i \varepsilon}$, respectively, as $\varepsilon \rightarrow 0$.

Remark 2.3. It is easy to show that the equilibrium $\left(u_{1}^{*}, u_{2}^{*}, \ldots, u_{n}^{*}\right)$ of $(2.1)$ is also unique if $\left(\mathrm{H}_{1}\right)-\left(\mathrm{H}_{4}\right)$ and the following $\left(\mathrm{H}_{5}\right)$ are satisfied.

The following well-known Barbalat lemma (see, e.g., [23]) will also be used.

Lemma 2.4. Let $f$ be a nonnegative function defined on $\mathbb{R}_{+}$such that $f$ is integrable and uniformly continuous on $\mathbb{R}_{+}$. Then $\lim _{t \rightarrow+\infty} f(t)=0$.

\section{Stability analysis}

In this section, we will consider the stability of the equilibrium $\left(u_{1}^{*}, u_{2}^{*}, \ldots, u_{n}^{*}\right)$ of system (2.1).

Let us first consider the case $t_{i j}^{(k)} \neq 0$, for some $i, j=1,2, \ldots, n, k=1,2, \ldots, K$. We further assume the following hypothesis.

$\left(\mathrm{H}_{5}\right)$ There exist positive constants $\lambda_{i}, i=1,2, \ldots, n$, such that the matrix:

$$
R=\left(\begin{array}{ccccc}
\eta_{1} & r_{12} & r_{13} & \cdots & r_{1 n} \\
r_{21} & \eta_{2} & r_{23} & \cdots & r_{2 n} \\
\vdots & \vdots & \vdots & \vdots & \vdots \\
r_{n 1} & r_{n 2} & r_{n 3} & \cdots & \eta_{n}
\end{array}\right)
$$


is negative definite, that is,

$$
(-1)^{i}\left(\begin{array}{ccccc}
\eta_{1} & r_{12} & r_{13} & \cdots & r_{1 n} \\
r_{21} & \eta_{2} & r_{23} & \cdots & r_{2 n} \\
\vdots & \vdots & \vdots & \vdots & \vdots \\
r_{n 1} & r_{n 2} & r_{n 3} & \cdots & \eta_{n}
\end{array}\right)>0, \quad i=1,2, \ldots, n
$$

where

$$
\begin{aligned}
\eta_{i}= & \lambda_{i}\left(-\frac{\underline{B_{i}}}{q_{i \varepsilon}}+\left(\sum_{k=0}^{K} t_{i i}^{(k)}\right)\right)+\frac{1}{2} \sum_{k=0}^{K} \sum_{j=1}^{n} \tau_{k}(t)\left[\frac{\lambda_{i} q_{j \varepsilon} \overline{B_{j}}}{p_{j \varepsilon}}\left|t_{i j}^{(k)}\right|+\frac{\lambda_{j} q_{i \varepsilon} \underline{B_{i}}}{p_{i \varepsilon}}\left|t_{j i}^{(k)}\right|\right] \\
& +\frac{1}{2} \sum_{k=0}^{K} \sum_{j=1}^{n} \tau_{k}(t)\left[\lambda_{i} q_{j \varepsilon}\left|t_{i j}^{(k)}\right|\left(\sum_{k=0}^{K} \sum_{l=1}^{n}\left|t_{j l}^{(k)}\right|\right)+q_{j \varepsilon}\left(\sum_{k=0}^{K} \sum_{l=1}^{n} \lambda_{l}\left|t_{l j}^{(k)}\right|\left|t_{j i}^{(k)}\right|\right)\right] \\
r_{i j}= & \frac{1}{2} \sum_{k=0}^{K}\left(\lambda_{i} t_{i j}^{(k)}+\lambda_{j} t_{j i}^{(k)}\right), \quad i \neq j, i, j=1,2, \ldots, n .
\end{aligned}
$$

Hence, the equilibrium $\left(u_{1}^{*}, u_{2}^{*}, \ldots, u_{n}^{*}\right)$ of system $(2.1)$ is unique.

Theorem 3.1. If $t_{i j}^{(k)} \neq 0$, for some $i, j=1,2, \ldots, n, k=1,2, \ldots, K$, and $\left(H_{1}\right)-\left(H_{5}\right)$ are satisfied, then the equilibrium $\left(u_{1}^{*}, u_{2}^{*}, \ldots, u_{n}^{*}\right)$ of system (2.1) is globally asymptotically stable.

Proof. Let

$$
\begin{aligned}
x_{j}(t) & =u_{j}(t)-u_{j}^{*}, \quad j=1,2, \ldots, n, \\
s_{j}\left(x_{j}(t)\right) & =f_{j}\left(x_{j}(t)+u_{j}^{*}\right)-f_{j}\left(u_{j}^{*}\right), \quad j=1,2, \ldots, n, \\
h_{j}\left(x_{j}(t)\right) & =b_{j}\left(x_{j}(t)+u_{j}^{*}\right)-b_{j}\left(u_{j}^{*}\right), \quad j=1,2, \ldots, n .
\end{aligned}
$$

Then, the stability properties of the equilibrium $\left(u_{1}^{*}, u_{2}^{*}, \ldots, u_{n}^{*}\right)$ of system $(2.1)$ are equivalent to that of the trivial solution of the following system:

$$
\dot{x}_{i}(t)=-a_{i}\left(x_{i}(t)+u_{i}^{*}\right)\left[h_{i}\left(x_{i}(t)\right)-\sum_{k=0}^{K} \sum_{j=1}^{n} t_{i j}^{(k)} s_{j}\left(x_{j}\left(t-\tau_{k}(t)\right)\right)\right] .
$$


We construct the following Lyapunov function:

$$
V_{1}=\sum_{i=1}^{n} \lambda_{i} \int_{0}^{x_{i}(t)} \frac{s_{i}(\xi)}{a_{i}\left(\xi+u_{i}^{*}\right)} d \xi
$$

Then its upper right Dini derivative is

$$
\begin{aligned}
\left.D^{+} V_{1}\right|_{(3.5)}= & \sum_{i=1}^{n} \lambda_{i} s_{i}\left(x_{i}(t)\right)\left[-h_{i}\left(x_{i}(t)\right)+\sum_{k=0}^{K} \sum_{j=1}^{n} t_{i j}^{(k)} s_{j}\left(x_{j}\left(t-\tau_{k}(t)\right)\right)\right] \\
= & \sum_{i=1}^{n} \lambda_{i} s_{i}\left(x_{i}(t)\right)\left[-h_{i}\left(x_{i}(t)\right)+\sum_{k=0}^{K} t_{i i}^{(k)} s_{j}\left(x_{j}(t)\right)\right] \\
& +\sum_{i=1}^{n} \lambda_{i} s_{i}\left(x_{i}(t)\right) \alpha_{i}+\sum_{k=0}^{K} \sum_{\substack { i=1 \\
\begin{subarray}{c}{j=1 \\
j \neq i{ i = 1 \\
\begin{subarray} { c } { j = 1 \\
j \neq i } }\end{subarray}}^{n} \lambda_{i} t_{i j}^{(k)} s_{i}\left(x_{i}(t)\right) s_{j}\left(x_{j}(t)\right),
\end{aligned}
$$

where

$$
\alpha_{i}=\sum_{k=0}^{K} \sum_{j=1}^{n} t_{i j}^{(k)} \int_{t}^{t-\tau_{k}(t)} s_{j}^{\prime}\left(x_{j}(\xi)\right) x_{j}^{\prime}(\xi) d \xi .
$$

Note that

$$
p_{i, \varepsilon} \equiv \min _{-\left(N_{i}+\mathcal{\varepsilon}\right) \leq w \leq N_{i}+\varepsilon} s_{i}^{\prime}(w) \leq \max _{-\left(N_{i}+\varepsilon\right) \leq w \leq N_{i}+\varepsilon} s_{i}^{\prime}(w) \equiv q_{i, \mathcal{\varepsilon}} .
$$

We also note that for sufficiently large $t$,

$$
\left|x_{i}(t)\right| \leq \frac{\left|s_{i}\left(x_{i}(t)\right)\right|}{p_{i \varepsilon}} .
$$

Then

$$
\begin{aligned}
\left|s_{i}\left(x_{i}(t)\right) \alpha_{i}\right| \leq & \left|s_{i}\left(x_{i}(t)\right)\right| \sum_{k=0}^{K} \sum_{j=1}^{n}\left|t_{i j}^{(k)}\right| \int_{t-\tau_{k}(t)}^{t}\left|s_{j}^{\prime}\left(x_{j}(\xi)\right)\right|\left|x_{j}^{\prime}(\xi)\right| d \xi \\
\leq & \left|s_{i}\left(x_{i}(t)\right)\right| \sum_{k=0}^{K} \sum_{j=1}^{n}\left|t_{i j}^{(k)}\right| \int_{t-\tau_{k}(t)}^{t} q_{j \varepsilon} \\
& \times\left(\left|h_{j}\left(x_{j}(\xi)\right)\right|+\sum_{k=0}^{K} \sum_{l=1}^{n}\left|t_{j l}^{(k)}\right| s_{l}\left(x_{l}\left(\xi-\tau_{k}(\xi)\right)\right)\right) d \xi
\end{aligned}
$$




$$
\begin{aligned}
\leq & \left|s_{i}\left(x_{i}(t)\right)\right| \sum_{k=0}^{K} \sum_{j=1}^{n}\left|t_{i j}^{(k)}\right| \int_{t-\tau_{k}(t)}^{t} q_{j \varepsilon} \\
& \times\left(\frac{\overline{B_{j}}}{p_{j \varepsilon}}\left|s_{j}\left(x_{j}(\xi)\right)\right|+\sum_{k=0}^{K} \sum_{l=1}^{n}\left|t_{j l}^{(k)}\right| s_{l}\left(x_{l}\left(\xi-\tau_{k}(\xi)\right)\right)\right) d \xi \\
\leq & \frac{1}{2} \sum_{k=0}^{K} \sum_{j=1}^{n}\left|t_{i j}^{(k)}\right| q_{j \varepsilon} \int_{t-\tau_{k}(t)}^{t} \\
& \times\left\{\frac{\overline{B_{j}}}{p_{j \varepsilon}}\left[s_{i}^{2}\left(x_{i}(t)\right)+s_{j}^{2}\left(x_{j}(t)\right)\right]\right. \\
= & \frac{1}{2} \sum_{k=0}^{K} \sum_{j=1}^{n}\left|t_{i j}^{(k)}\right| q_{j \varepsilon} \tau_{k}(t)\left[\frac{B_{j}}{p_{j \varepsilon}}+\sum_{k=0}^{K} \sum_{l=1}^{n}\left|t_{j l}^{(k)}\right|\right] s_{i}^{2}\left(x_{l}(t)\right) \\
& \left.+\frac{1}{2} \sum_{k=0}^{K} \sum_{j=1}^{n}\left|t_{i j}^{(k)}\right| \int_{t-\tau_{k}(t)}^{t} q_{j \varepsilon}^{(k)} \mid\left[s_{i}^{2}\left(x_{i}(t)\right)+s_{l}^{2}\left(x_{l}\left(\xi-\tau_{k}(\xi)\right)\right)\right]\right\} d \xi \\
& \times\left[\frac{B_{j}}{p_{j \varepsilon}} s_{j}^{2}\left(x_{j}(\xi)\right)+\sum_{k=0}^{K} \sum_{l=1}^{n}\left|t_{j l}^{(k)}\right| s_{l}^{2}\left(x_{l}\left(\xi-\tau_{k}(\xi)\right)\right)\right] d \xi \\
= & \sum_{k=0}^{K} \sum_{j=1}^{n}\left|t_{i j}^{(k)}\right|\left(A_{j k \varepsilon} s_{i}^{2}\left(x_{i}(t)\right)+\int_{t-\tau_{k}(t)}^{t} \mu_{j \varepsilon}(\xi) d \xi\right),
\end{aligned}
$$

where

$$
\begin{aligned}
A_{j k \varepsilon} & =\frac{1}{2} q_{j \varepsilon} \tau_{k}(t)\left[\frac{\overline{B_{j}}}{p_{j \varepsilon}}+\sum_{k=0}^{K} \sum_{l=1}^{n}\left|t_{j l}^{(k)}\right|\right], \\
\mu_{j \varepsilon}(\xi) & =\frac{1}{2} q_{j \varepsilon}\left[\frac{\overline{B_{j}}}{p_{j \varepsilon}} s_{j}^{2}\left(x_{j}(\xi)\right)+\sum_{k=0}^{K} \sum_{l=1}^{n}\left|t_{j l}^{(k)}\right| s_{l}^{2}\left(x_{l}\left(\xi-\tau_{k}(\xi)\right)\right)\right] .
\end{aligned}
$$

Furthermore, by $\left(\mathrm{H}_{1}\right)$, we have for $t \geq T+\Delta$ that

$$
\begin{aligned}
& s_{i}\left(x_{i}(t)\right)\left[-h_{i}\left(x_{i}(t)\right)+\sum_{k=0}^{K} t_{i i}^{(k)} s_{i}\left(x_{i}(t)\right)\right] \\
& \quad \leq-\underline{B_{i}} x_{i}(t) f_{i}\left(x_{i}(t)\right)+\left(\sum_{k=0}^{K} t_{i i}^{(k)}\right) s_{i}^{2}\left(x_{i}(t)\right) \leq\left[-\frac{B_{i}}{q_{i \varepsilon}}+\left(\sum_{k=0}^{K} t_{i i}^{(k)}\right)\right] s_{i}^{2}\left(x_{i}(t)\right) .
\end{aligned}
$$


Let

$$
V_{2}=\sum_{k=0}^{K} \sum_{i=1}^{n} \sum_{j=1}^{n} \lambda_{i}\left|t_{i j}^{(k)}\right|\left(\int_{t-\tau_{k}(t)}^{t} \int_{\theta}^{t} \mu_{j \varepsilon}(\xi) d \xi d \theta+\frac{\tau_{k}(t) q_{j \varepsilon}}{2} \sum_{k=0}^{K} \sum_{l=1}^{n}\left|t_{j l}^{(k)}\right| \int_{t-\tau_{k}(t)}^{t} s_{l}^{2}\left(x_{l}(\xi)\right) d \xi\right) .
$$

Its derivative is

$$
\begin{aligned}
\left.D^{+} V_{2}\right|_{(3.5)}= & \sum_{k=0}^{K} \sum_{i=1}^{n} \sum_{j=1}^{n} \frac{\lambda_{i} q_{j \varepsilon}}{2}\left|t_{i j}^{(k)}\right| \tau_{k}(t)\left(\frac{\overline{B_{j}}}{p_{j \varepsilon}} s_{j}^{2}\left(x_{j}(t)\right)+\sum_{k=0}^{K} \sum_{l=1}^{n}\left|t_{j l}^{(k)}\right| s_{l}^{2}\left(x_{l}(t)\right)\right) \\
& -\sum_{k=0}^{K} \sum_{i=1}^{n} \sum_{j=1}^{n} \lambda_{i}\left|t_{i j}^{(k)}\right| \int_{t-\tau_{k}(t)}^{t} \mu_{j \varepsilon}(\xi) d \xi .
\end{aligned}
$$

Hence,

$$
\begin{aligned}
D^{+} V= & D^{+} V_{1}+D^{+} V_{2} \\
\leq & \sum_{i=1}^{n} \lambda_{i}\left[-\frac{B_{i}}{q_{i \varepsilon}}+\left(\sum_{k=0}^{K} t_{i i}^{(k)}\right)\right] s_{i}^{2}\left(x_{i}(t)\right)+\sum_{k=0}^{K} \sum_{i=1}^{n} \sum_{j=1}^{n} \lambda_{i}\left|t_{i j}^{(k)}\right| A_{j k \varepsilon} s_{i}^{2}\left(x_{i}(t)\right) \\
& +\sum_{k=0}^{K} \sum_{i=1}^{n} \sum_{j=1}^{n} \lambda_{i} t_{i j}^{(k)} s_{i}\left(x_{i}(t)\right) s_{j}\left(x_{j}(t)\right) \\
& +\sum_{k=0}^{K} \sum_{i=1}^{n} \sum_{j=1}^{n} \frac{\lambda_{i} q_{j \varepsilon}}{2}\left|t_{i j}^{(k)}\right| \tau_{k}(t)\left(\frac{\overline{B_{j}}}{p_{j \varepsilon}} s_{j}^{2}\left(x_{j}(t)\right)+\sum_{k=0}^{K} \sum_{l=1}^{n}\left|t_{j l}^{(k)}\right| s_{l}^{2}\left(x_{l}(t)\right)\right) \\
=\sum_{i=1}^{n} & \lambda_{i}\left[-\frac{B_{i}}{q_{i \varepsilon}}+\left(\sum_{k=0}^{K} t_{i i}^{(k)}\right)+\sum_{k=0}^{K} \sum_{j=1}^{n}\left|t_{i j}^{(k)}\right| A_{j k \varepsilon}\right] s_{i}^{2}\left(x_{i}(t)\right) \\
& +\sum_{k=0}^{K} \sum_{i=1}^{n} \sum_{j=1}^{n} \lambda_{i} t_{i j}^{(k)} s_{i}\left(x_{i}(t)\right) s_{j}\left(x_{j}(t)\right) \\
& +\sum_{k=0}^{K} \sum_{i=1}^{n} \sum_{i=1}^{n} \sum_{j=1}^{n} \frac{\lambda_{i} q_{j \varepsilon}}{2}\left|t_{j i}^{(k)}\right| \tau_{k}(t) \frac{\underline{B}_{j}}{p_{i \varepsilon}} s_{i}^{2}\left(x_{i}(t)\right) \\
& \left.\sum_{k=0}^{K} \sum_{l=1}^{n} \frac{\lambda_{l} q_{j \varepsilon}}{2}\left|t_{l j}^{(k)}\right| \tau_{k}(t)\left|t_{j i}^{(k)}\right| s_{i}^{2}\left(x_{i}(t)\right)\right)
\end{aligned}
$$




$$
\begin{aligned}
& =\sum_{i=1}^{n}\left\{\lambda_{i}\left[-\frac{\underline{B_{i}}}{q_{i \varepsilon}}+\left(\sum_{k=0}^{K} t_{i i}^{(k)}\right)+\sum_{k=0}^{K} \sum_{j=1}^{n}\left|t_{i j}^{(k)}\right| \frac{q_{j \varepsilon} \tau_{k}(t)}{2}\left(\frac{\overline{B_{j}}}{p_{j \varepsilon}}+\sum_{k=0}^{K} \sum_{l=1}^{n}\left|t_{j l}^{(k)}\right|\right)\right]\right. \\
& +\frac{q_{i \varepsilon} \underline{B_{i}}}{2 p_{i \varepsilon}} \sum_{k=0}^{K} \sum_{j=1}^{n} \lambda_{j}\left|t_{j i}^{(k)}\right| \tau_{k}(t) \\
& \left.+\sum_{k=0}^{K} \sum_{j=1}^{n} \frac{q_{j \varepsilon}}{2}\left(\sum_{k=0}^{K} \sum_{l=1}^{n} \lambda_{l} \tau_{k}(t)\left|t_{l j}^{(k)}\right|\left|t_{j i}^{(k)}\right|\right)\right\} s_{i}^{2}\left(x_{i}(t)\right) \\
& +\sum_{k=0}^{K} \sum_{i=1}^{n} \sum_{j=1}^{n} \lambda_{i} t_{i j}^{(k)} s_{i}\left(x_{i}(t)\right) s_{j}\left(x_{j}(t)\right) \\
& =\sum_{i=1}^{n}\left\{\lambda_{i}\left(-\frac{\underline{B_{i}}}{q_{i \varepsilon}}+\left(\sum_{k=0}^{K} t_{i i}^{(k)}\right)\right)+\frac{1}{2} \sum_{k=0}^{K} \sum_{j=1}^{n}\left[\frac{\lambda_{i} q_{j \varepsilon} \overline{B_{j}}}{p_{j \varepsilon}}\left|t_{i j}^{(k)}\right| \tau_{k}(t)+\frac{\lambda_{j} q_{i \varepsilon} \underline{B_{i}}}{p_{i \varepsilon}}\left|t_{j i}^{(k)}\right| \tau_{k}(t)\right]\right. \\
& +\frac{1}{2} \sum_{k=0}^{K} \sum_{j=1}^{n}\left[\lambda_{i} q_{j \varepsilon} \tau_{k}(t)\left|t_{i j}^{(k)}\right|\left(\sum_{k=0}^{K} \sum_{l=1}^{n}\left|t_{j l}^{(k)}\right|\right)\right. \\
& \left.\left.+q_{j \varepsilon} \tau_{k}(t)\left(\sum_{k=0}^{K} \sum_{l=1}^{n} \lambda_{l}\left|t_{l j}^{(k)}\right|\left|t_{j i}^{(k)}\right|\right)\right]\right\} s_{i}^{2}\left(x_{i}(t)\right) \\
& +\sum_{k=0}^{K} \sum_{i=1}^{n} \sum_{j=1}^{n} \lambda_{i} t_{i j}^{(k)} s_{i}\left(x_{i}(t)\right) s_{j}\left(x_{j}(t)\right) \\
& =\frac{1}{2}\left(s_{1}\left(x_{1}(t)\right), s_{2}\left(x_{2}(t)\right), \ldots, s_{n}\left(x_{n}(t)\right)\right) R(\varepsilon)\left(s_{1}\left(x_{1}(t)\right), s_{2}\left(x_{2}(t)\right), \ldots, s_{n}\left(x_{n}(t)\right)\right)^{T} \text {, }
\end{aligned}
$$

where

$$
\begin{aligned}
R(\varepsilon)= & \left(\begin{array}{ccccc}
\eta_{1}(\varepsilon) & r_{12} & r_{13} & \cdots & r_{1 n} \\
r_{21} & \eta_{2}(\varepsilon) & r_{23} & \cdots & r_{2 n} \\
\vdots & \vdots & \vdots & \vdots & \vdots \\
r_{n 1} & r_{n 2} & r_{n 3} & \cdots & \eta_{n}(\varepsilon)
\end{array}\right), \\
\eta_{i}= & \lambda_{i}\left(-\frac{B_{i}}{q_{i \varepsilon}}+\left(\sum_{k=0}^{K} t_{k k}^{(k)}\right)\right)+\frac{1}{2} \sum_{k=0}^{K} \sum_{j=1}^{n} \tau_{k}(t)\left[\frac{\lambda_{i} q_{j \varepsilon} \overline{B_{j}}}{p_{j \varepsilon}}\left|t_{i j}^{(k)}\right|+\frac{\lambda_{j} q_{i \varepsilon} B_{i}}{p_{i \varepsilon}}\left|t_{j i}^{(k)}\right|\right] \\
& +\frac{1}{2} \sum_{k=0}^{K} \sum_{j=1}^{n} \tau_{k}(t)\left[\lambda_{i} q_{j \varepsilon}\left|t_{i j}^{(k)}\right|\left(\sum_{k=0}^{K} \sum_{l=1}^{n}\left|t_{i l}^{(k)}\right|\right)+q_{j \varepsilon}\left(\sum_{k=0}^{K} \sum_{l=1}^{n} \lambda_{l}\left|t_{l j}^{(k)}\right|\left|t_{j i}^{(k)}\right|\right)\right] \\
r_{i j}= & \frac{1}{2} \sum_{k=0}^{K}\left(\lambda_{i} t_{i j}^{(k)}+\lambda_{j} t_{j i}^{(k)}\right), \quad i \neq j, i, j=1,2, \ldots, n .
\end{aligned}
$$


Moreover, the upper right derivative $D^{+} V$ of $V$ along solution (3.5) satisfies

$$
\left.D^{+} V\right|_{(3.5)} \leq-\delta(\varepsilon) \sum_{i=1}^{n} s_{i}^{2}\left(x_{i}(t)\right)
$$

for $t \geq T+\bar{\tau}$. Here $\delta(\varepsilon)>0$ is some constant. $x_{t}=x(t+s)$ for $-\bar{\tau} \leq s \leq 0$.

Integrating (3.18) over $[T+\bar{\tau}, t]$ yields

$$
V\left(x_{t}\right)+\delta(\varepsilon) \int_{T+\bar{\tau}}^{t} \sum_{i=1}^{n} s_{i}^{2}\left(x_{i}(\xi)\right) d \xi \leq V\left(x_{T+\bar{\tau}}\right),
$$

which implies that

$$
\sum_{i=1}^{n} \int_{0}^{+\infty} s_{i}^{2}\left(x_{i}(\xi)\right) d \xi<+\infty .
$$

Moreover, from Lemma 2.2 and $\left(\mathrm{H}_{2}\right)-\left(\mathrm{H}_{3}\right)$, we see that $s_{i}^{2}\left(x_{i}(t)\right), i=1,2, \ldots, n$, are also uniformly continuous on $\mathbb{R}_{+}$. Hence, Lemma 2.4 implies that

$$
\lim _{t \rightarrow+\infty}\left|s_{i}\left(x_{i}(t)\right)\right|=0, \quad i=1,2, \ldots, n .
$$

Again from Lemma 2.2 and $\left(\mathrm{H}_{2}\right)$, we have

$$
\lim _{t \rightarrow+\infty} x_{i}(t)=0, \quad i=1,2, \ldots, n,
$$

that is,

$$
\lim _{t \rightarrow+\infty} u_{i}(t)=u_{i}^{*}, \quad i=1,2, \ldots, n,
$$

which show that the equilibrium $\left(u_{1}^{*}, u_{2}^{*}, \ldots, u_{n}^{*}\right)$ of system $(2.1)$ is globally attractive.

Furthermore, note $\left(\mathrm{H}_{2}\right),\left(\mathrm{H}_{3}\right)$, and the following inequalities:

$$
p_{i} \leq f_{i}^{\prime}\left(u_{i}^{*}\right) \leq q_{i}, \quad i=1,2, \ldots, n .
$$

We see that $\left(\mathrm{H}_{5}\right)$ implies $\left(\mathrm{H}_{6}\right)$ of Theorem 3.4. Thus, the equilibrium $\left(u_{1}^{*}, u_{2}^{*}, \ldots, u_{n}^{*}\right)$ of system (2.1) is also locally asymptotically stable. This proves Theorem 3.1.

$\left(\mathrm{H}_{5}^{\prime}\right)$ There exist positive constants $\lambda_{i}, i=1,2, \ldots, n$, such that

$$
\begin{aligned}
\gamma_{i}= & \lambda_{i}\left(-\frac{\underline{B_{i}}}{q_{i \varepsilon}}+\left(\sum_{k=0}^{K} t_{i i}^{(k)}\right)\right)+\frac{1}{2} \sum_{k=0}^{K} \sum_{j=1}^{n} \tau_{k}(t)\left[\frac{\lambda_{i} q_{j} \overline{B_{j}}}{p_{j}}\left|t_{i j}^{(k)}\right|+\frac{\lambda_{j} q_{i} \underline{B_{i}}}{p_{i}}\left|t_{j i}^{(k)}\right|\right] \\
& +\frac{1}{2} \sum_{k=0}^{K} \sum_{j=1}^{n} \tau_{k}(t)\left[\lambda_{i} q_{j}\left|t_{i j}^{(k)}\right|\left(\sum_{k=0}^{K} \sum_{l=1}^{n}\left|t_{j l}^{(k)}\right|\right)+q_{j}\left(\sum_{k=0}^{K} \sum_{l=1}^{n} \lambda_{l}\left|t_{l j}^{(k)}\right|\left|t_{j i}^{(k)}\right|\right)\right] \\
& +\frac{1}{2} \sum_{k=0}^{K}\left[\lambda_{i}\left|t_{i j}^{(k)}\right|+\lambda_{j}\left|t_{j i}^{(k)}\right|\right]<0 .
\end{aligned}
$$

By the process of the proof of Theorem 3.1, we can easily obtain the following. 
Corollary 3.2. If $t_{i j}^{(k)} \neq 0$, for some $i, j=1,2, \ldots, n$, and $\left(H_{1}\right)-\left(H_{4}\right)$ and $\left(H_{5}^{\prime}\right)$ are satisfied, then the equilibrium $\left(u_{1}^{*}, u_{2}^{*}, \ldots, u_{n}^{*}\right)$ of system (2.1) is also globally asymptotically stable.

Remark 3.3. In $[11,14,18,24]$, the authors require that the time-varying delays satisfy $\tau_{k}^{\prime}(t) \leq R<1$. However, in our theorem, these delays are not necessarily continuous and differentiable. They only need to satisfy the condition $0 \leq \tau_{k}(t) \leq \bar{\tau}$. Hence, our results are less restrictive and conservative than the known result $[7,19]$.

$\left(\mathrm{H}_{6}\right)$ There exist positive constants $\lambda_{i}, i=1,2, \ldots, n$, such that the matrix

$$
R^{*}=\left(\begin{array}{ccccc}
\eta_{1}^{*} & r_{12} & r_{13} & \cdots & r_{1 n} \\
r_{21} & \eta_{2}^{*} & r_{23} & \cdots & r_{2 n} \\
\vdots & \vdots & \vdots & \vdots & \vdots \\
r_{n 1} & r_{n 2} & r_{n 3} & \cdots & \eta_{n}^{*}
\end{array}\right)
$$

is negative definite, where

$$
\begin{aligned}
\eta_{i}^{*}= & \lambda_{i}\left(-\frac{B_{i}}{f_{i}^{\prime}\left(u_{i}^{*}\right)}+\left(\sum_{k=0}^{K} t_{i i}^{(k)}\right)\right)+\frac{1}{2} \sum_{k=0}^{K} \sum_{j=1}^{n} \tau_{k}(t)\left[\lambda_{i} \overline{B_{j}}\left|t_{i j}^{(k)}\right|+\lambda_{j} \underline{B}_{j}\left|t_{i j}^{(k)}\right|\right] \\
& +\frac{1}{2} \sum_{k=0}^{K} \sum_{j=1}^{n} \tau_{k}(t)\left[\lambda_{i} f_{j}^{\prime}\left(u_{j}^{*}\right)\left|t_{i j}^{(k)}\right|\left(\sum_{k=0}^{K} \sum_{l=1}^{n}\left|t_{j l}^{(k)}\right|\right)+f_{j}^{\prime}\left(u_{j}^{*}\right)\left(\sum_{k=0}^{K} \sum_{l=1}^{n} \lambda_{l}\left|t_{l j}^{(k)}\right|\left|t_{j i}^{(k)}\right|\right)\right] \\
r_{i j}= & \frac{1}{2} \sum_{k=0}^{K}\left(\lambda_{i} t_{i j}^{(k)}+\lambda_{j} t_{j i}^{(k)}\right), \quad i \neq j, i, j=1,2, \ldots, n .
\end{aligned}
$$

Hence, we can easily have the following.

Theorem 3.4. If $t_{i j}^{(k)} \neq 0$, for some $i, j=1,2, \ldots, n, k=1,2, \ldots, K$, and $\left(H_{1}\right)-\left(H_{4}\right)$ and $\left(H_{6}\right)$ are satisfied, then the equilibrium $\left(u_{1}^{*}, u_{2}^{*}, \ldots, u_{n}^{*}\right)$ of system $(2.1)$ is also locally asymptotically stable.

\section{Numerical example and conclusions}

Generally, the delay-independent criteria are particularly restrictive and conservative for networks parameters. Moreover, it is reasonable to consider and apply these criteria first. If they are found inappropriate, the delay-dependent criteria will then be applied. To illustrate the results presented in Theorem 3.1 and Corollary 3.2, a simple example is given and a comparison of the results is given based on the results of literature [7] in the following.

We consider the following model system:

$$
\begin{aligned}
& \dot{x}_{1}(t)=-\left(4+\sin \left(x_{1}(t)\right)\right)\left[2 x_{1}(t)-\tanh \left(x_{1}(t)\right)-0.5 \tanh \left(2 x_{2}(t-\tau)\right)+0.5\right], \\
& \dot{x}_{2}(t)=-\left(2+\cos \left(x_{2}(t)\right)\right)\left[2 x_{2}(t)-\tanh \left(x_{1}(t)\right)-0.5 \tanh \left(2 x_{2}(t-\tau)\right)-0.5\right] .
\end{aligned}
$$




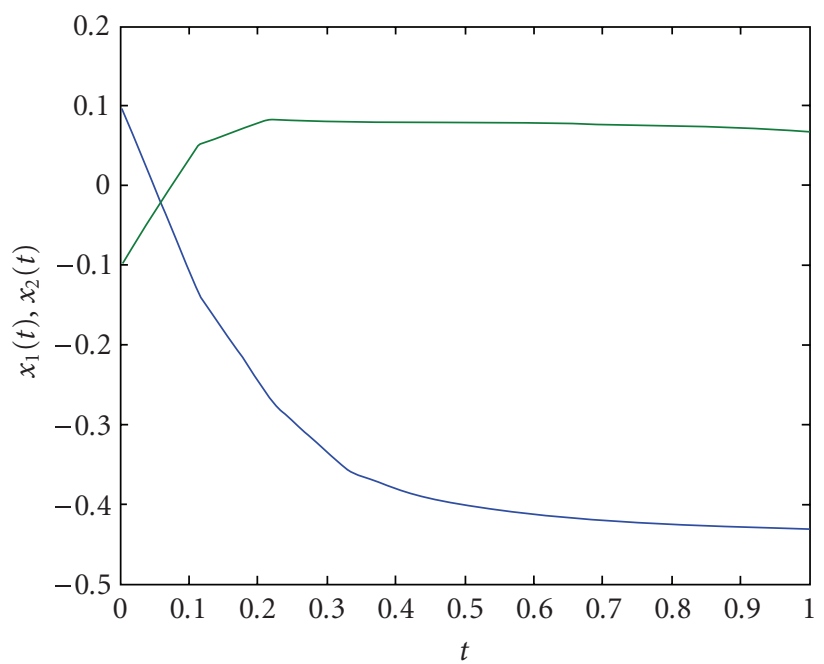

Figure 4.1. Wave form plot for system (4.1) when $\tau=0.282$.

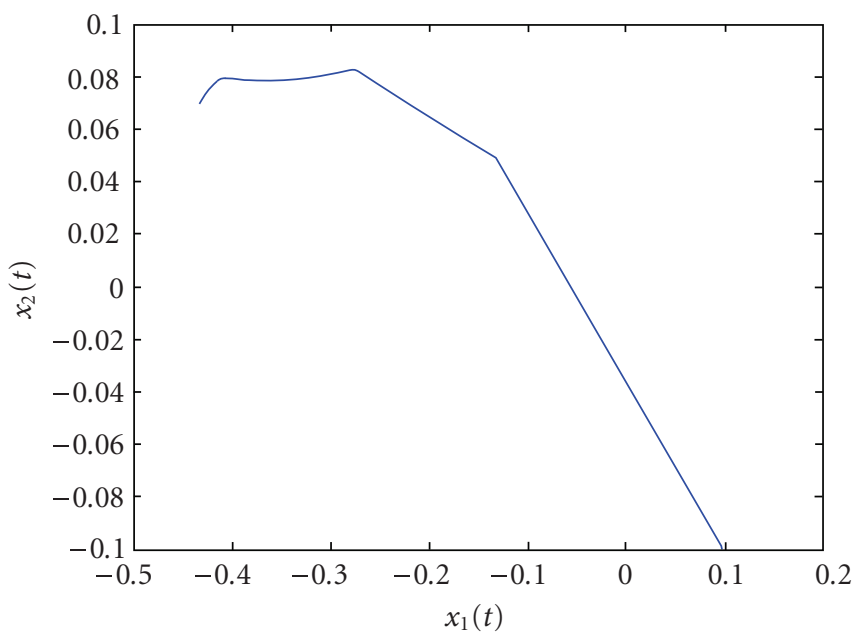

Figure 4.2. Phase plane plot for system (4.1) when $\tau=0.282$.

We can easily find that the delay-independent conditions given in [7] are not applied and satisfied. This demonstrates that the delay-independent criteria are more conservative and restrictive than the delay-dependent criteria.

For system (4.1), we can obtain $\tau<0.2828$ from [7, Theorem 3.1]. However, we can also obtain $\tau \leq 0.8246$ based on our results of Theorem 3.1. Numerical simulations have also been performed (see Figures 4.1, 4.2, 4.3 and 4.4). However, the problem of whether the delay superbound is optimal will be studied in a forthcoming paper.

In this paper, we have analyzed Cohen-Grossberg model with time delays in detail. The global asymptotic stability criteria for the equilibrium are derived based on the approach of Lyapunov functional. The obtained results are delay-dependent. Then, the 


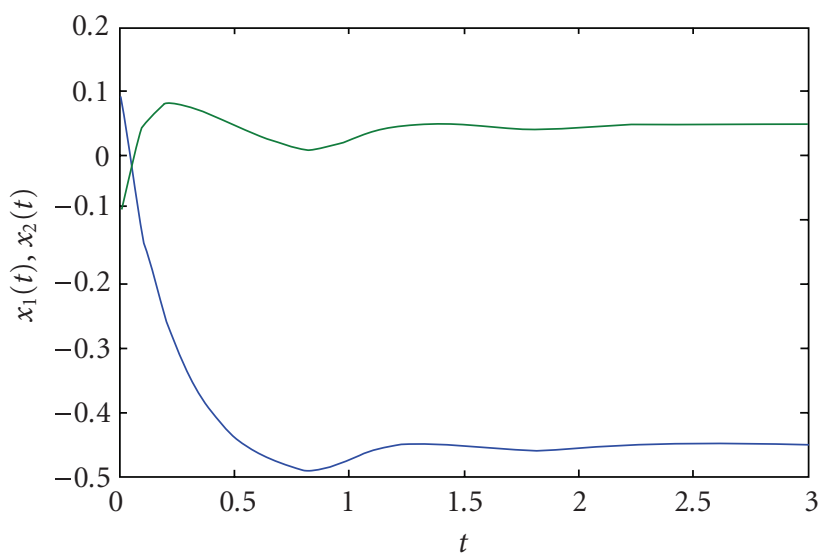

Figure 4.3. Wave form plot for system (4.1) when $\tau=0.8246$.

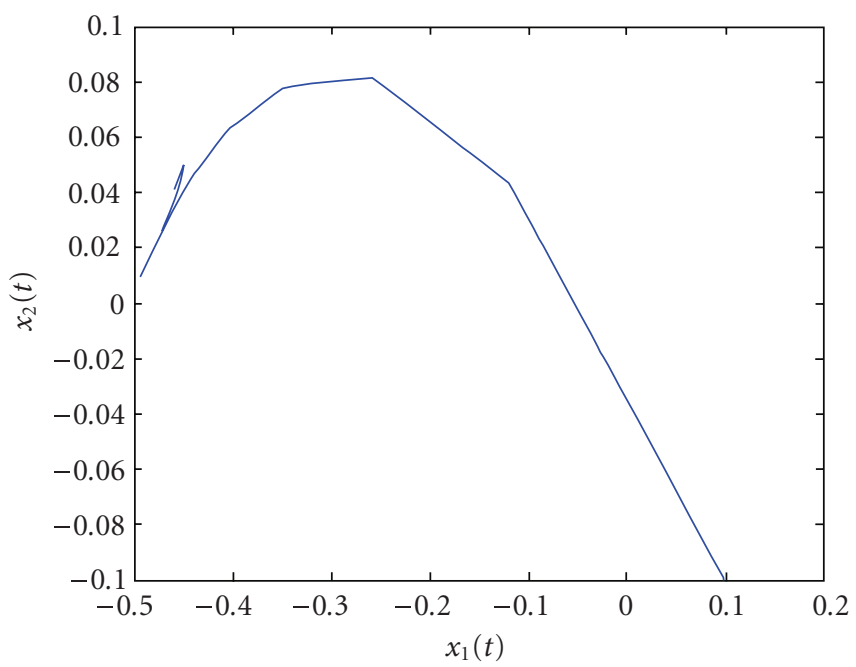

Figure 4.4. Phase plane plot for system (4.1) when $\tau=0.8246$.

delay-dependent criteria for local asymptotic stability criteria have also been obtained. Hence, our work has complemented and generalized that reported in [7].

\section{Acknowledgments}

The work described in this paper was supported by grants from the National Natural Science Foundation of China (no. 60573047), Program for New Century Excellent Talents in University, the Doctorate Foundation Grants from the Ministry of Education of China (no. 20020611007), and the Natural Science Foundation of Chongqing (no. 8509, 8986-3). 


\section{References}

[1] M. A. Cohen and S. Grossberg, "Absolute stability of global pattern formation and parallel memory storage by competitive neural networks," IEEE Transactions on Systems, Man, and Cybernetics, vol. 13, no. 5, pp. 815-826, 1983.

[2] S. Grossberg, "Nonlinear neural networks: principles, mechanisms, and architectures," Neural Networks, vol. 1, no. 1, pp. 17-61, 1988.

[3] J. J. Hopfield, "Neural networks and physical systems with emergent collective computational abilities," Proceedings of the National Academy of Sciences of the United States of America, vol. 79, no. 8, pp. 2554-2558, 1982.

[4] J. J. Hopfield, "Neurons with graded response have collective computational properties like those of two-state neurons," Proceeding of the National Academy of Sciences, vol. 81, no. 10, pp. 30883092, 1984.

[5] X. Liao, K. W. Wong, and J. B. Yu, "Novel stability conditions for cellular neural networks with time delay," International Journal of Bifurcation and Chaos, vol. 11, no. 7, pp. 1853-1864, 2001.

[6] X. Liao, Z. Wu, and J. Yu, "Stability analyses of cellular neural networks with continuous time delay," Journal of Computational and Applied Mathematics, vol. 143, no. 1, pp. 29-47, 2002.

[7] H. Ye, A. N. Michel, and K. Wang, "Qualitative analysis of Cohen-Grossberg neural networks with multiple delays," Physical Review E, vol. 51, no. 3, pp. 2611-2618, 1995.

[8] P. van den Driessche and X. Zou, "Global attractivity in delayed Hopfield neural network models," SIAM Journal on Applied Mathematics, vol. 58, no. 6, pp. 1878-1890, 1998.

[9] C. M. Marcus and R. M. Westervelt, "Stability of analog neural networks with delay," Physical Review. A, vol. 39, no. 1, pp. 347-359, 1989.

[10] K. Gopalsamy and X. Z. He, "Stability in asymmetric Hopfield nets with transmission delays," Physica D, vol. 76, no. 4, pp. 344-358, 1994.

[11] M. Joy, "Results concerning the absolute stability of delayed neural networks," Neural Networks, vol. 13, no. 6, pp. 613-616, 2000.

[12] X. Liao and J. B. Yu, "Robust stability for interval Hopfield neural networks with time delay," IEEE Transactions on Neural Networks, vol. 9, no. 5, pp. 1042-1045, 1998.

[13] X. Liao, K. W. Wong, Z. Wu, and G. Chen, "Novel robust stability criteria for interval-delayed Hopfield neural networks," IEEE Transactions on Circuits and Systems. I, vol. 48, no. 11, pp. 1355-1359, 2001.

[14] X. Liao, G. Chen, and E. N. Sanchez, "LMI-based approach for asymptotically stability analysis of delayed neural networks," IEEE Transactions on Circuits and Systems. I, vol. 49, no. 7, pp. 1033-1039, 2002.

[15] K. Gopalsamy and X. Z. He, "Delay-independent stability in bi-directional associative memory networks," IEEE Transactions on Neural Networks, vol. 5, no. 6, pp. 998-1002, 1994.

[16] V. Sree Hari Rao and Bh. R. M. Phaneendra, "Global dynamics of bi-directional associative memory neural networks involving transmission delays and dead zones," Neural Networks, vol. 12, no. 3, pp. 455-465, 1999.

[17] X. Liao and J. B. Yu, "Qualitative analysis of bi-directional associative memory networks with time delays," International Journal of Circuit Theory and Applications, vol. 26, no. 3, pp. 219-229, 1998.

[18] X. Liao, J. Yu, and G. Chen, "Novel stability criteria for bi-directional associative memory neural networks with time delays," International Journal of Circuit Theory and Applications, vol. 30, no. 5, pp. 519-546, 2002.

[19] L. Wang and X. Zou, "Harmless delays in Cohen-Grossberg neural networks," Physica D, vol. 170, no. 2, pp. 162-173, 2002.

[20] X. Liao, K. W. Wong, and C. G. Li, "Global exponential stability for a class of generalized neural networks with distributed delays," Nonlinear Analysis: Real World Applications, vol. 5, no. 3, pp. 527-547, 2004. 
[21] X. Liao, C. G. Li, and K. W. Wong, "Criteria for exponential stability of Cohen-Grossberg neural networks," Neural Networks, vol. 17, no. 10, pp. 1401-1414, 2004.

[22] J. K. Hale and S. M. Verduyn Lunel, Introduction to Functional-Differential Equations, vol. 99 of Applied Mathematical Sciences, Springer, New York, NY, USA, 1993.

[23] K. Gopalsamy, Stability and Oscillations in Delay Differential Equations of Population Dynamics, vol. 74 of Mathematics and Its Applications, Kluwer Academic Publishers, Dordrecht, The Netherlands, 1992.

[24] X. Liao, G. Chen, and E. N. Sanchez, "Delay-dependent exponential stability analysis of delayed neural networks: an LMI approach,” Neural Networks, vol. 15, no. 7, pp. 855-866, 2002.

Xiaofeng Liao: School of Computer Science and Information, Chongqing Jiaotong University, Chongqing 400074, China; Department of Computer Science and Engineering,

Chongqing University, Chongqing 400044, China

Email address: xfliao@cqu.edu.cn

Songtao Guo: Department of Computer Science and Engineering, Chongqing University,

Chongqing 400044, China

Email address: songtao@cqu.edu.cn 


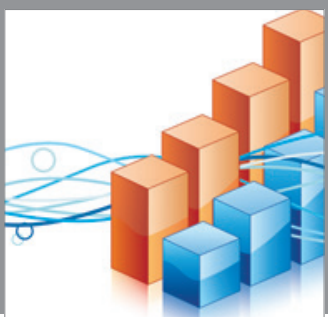

Advances in

Operations Research

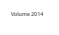

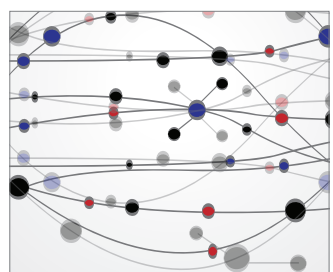

\section{The Scientific} World Journal
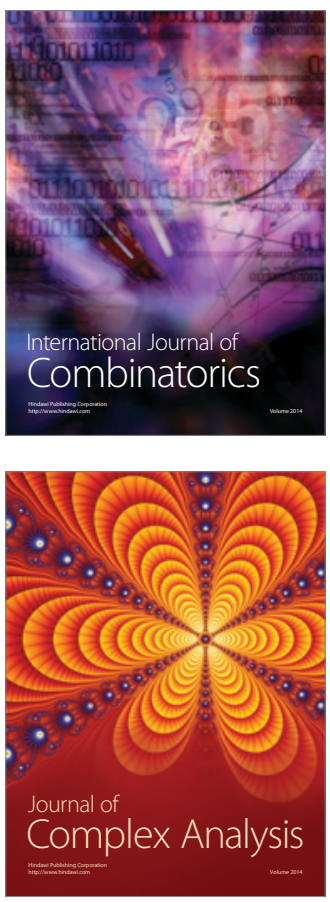

International Journal of

Mathematics and

Mathematical

Sciences
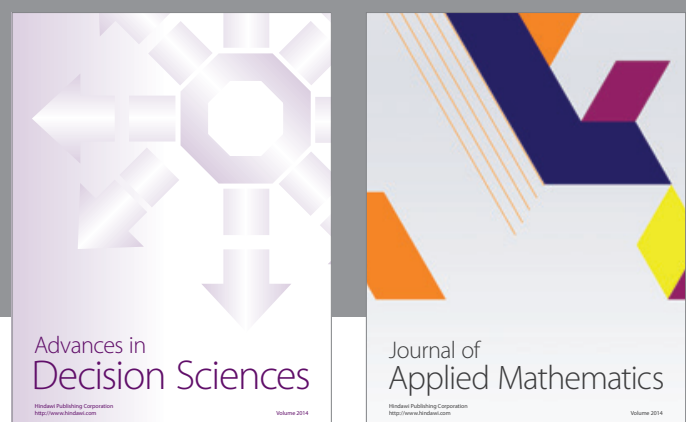

Journal of

Applied Mathematics
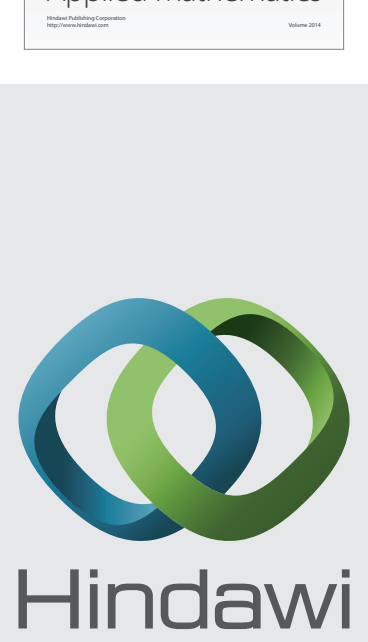

Submit your manuscripts at http://www.hindawi.com
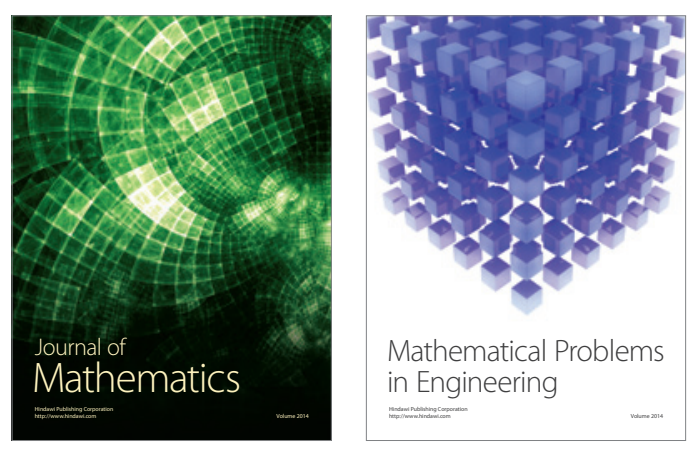

Mathematical Problems in Engineering
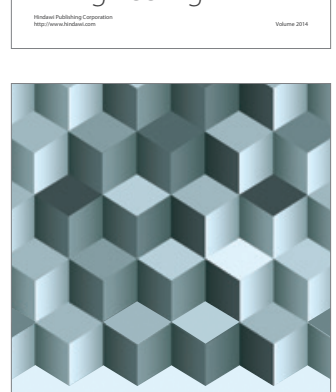

Journal of

Function Spaces
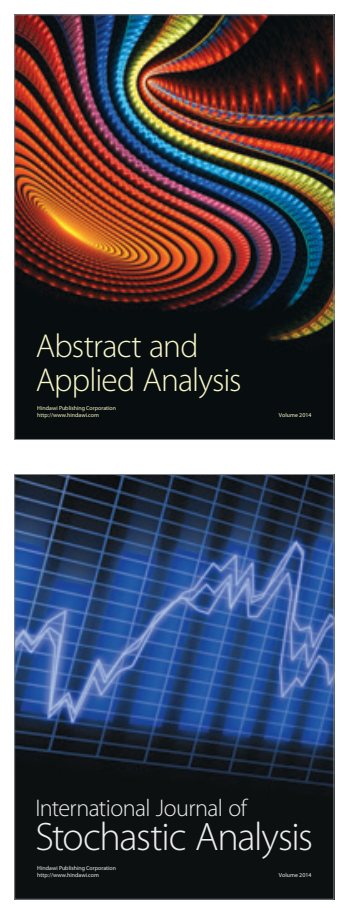

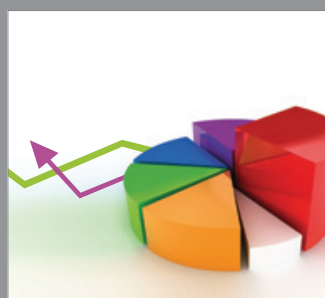

ournal of

Probability and Statistics

Promensencen
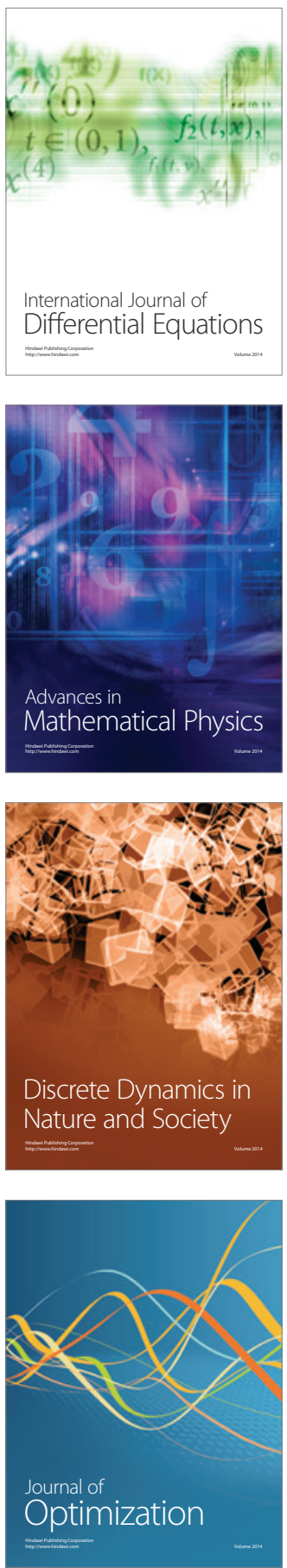